\title{
Research on Mechanical Braking Model of Cows Knee Joint
}

\author{
Meng Liu ${ }^{1, \dagger}$, Kexin Meng ${ }^{2, \dagger}$, Shuli Mei ${ }^{3, \dagger, *}$, and Ruyi Xing ${ }^{4, \dagger}$ \\ 1 College of Information and Electrical Engineering, China Agricultural University; bynd_lm@163.com \\ 2 College of Information and Electrical Engineering, China Agricultural University; 17853516390@163.com \\ 3 College of Information and Electrical Engineering, China Agricultural University; meishuli@ 163.com \\ 4 Center of education technology, Heibei University of Engineering; xingruyi_hue@163.com \\ * Correspondence: meishuli@ 163.com \\ $\dagger$ These authors contributed equally to this work.
}

Abstract: The shape of the knee is a chain-like structure of an ellipsoid. This suggests that, in the latest study, in addition to rolling, there is slippage in the motion of the knee. This paper selects a mechanical braking model for cattle based on two mechanical structures in different directions at the joints. The experimental results show that the modified dynamical system has strong chaotic properties. This can be one of the basis for judging various health states.

\section{Introduction}

Farmers make the best use of their cows and feed them economically while maintaining health for maximum yield and milk quality per cow with minimal investment. The initial symptoms of common diseases in dairy cows are usually multi-characteristic, and changes in excitability and posture are reflected in changes in gait, and changes in weight and gait lead to changes in the signal curve obtained by the pressure sensor [1]. It is not difficult to know that the low frequency component of the pressure sensor's signal can be used to measure body weight. The high frequency compressive stress of the bovine information signal can reflect the temperature, excitation and method of bovine, which can be used to analyze the early symptoms of common diseases [2]. The researchers found the existence of [3] bifurcations in the dynamic system of crows. In general, the high frequency component of the pressure sensor signal should be a chaotic signal, not a periodic signal measured from healthy cows, instead, for unhealthy cows, such as lame cows, the periodic component introduced by periodic lameness will overcome confusion. Therefore, the chaotic dynamic leg model of cows helps to identify various unhealthy cows through a weighting system, as shown in Figure 1.

\section{Construction of the chaotic dynamitic system}

The duplex model shown in Figure 2 (a) is a common beam support feeding system. This is a deterministic system that does not cause confusion, as it does not involve sliding. Long [4] proposed a dynamic three-link model, as shown in Figure 2 (b), which claims to produce chaotic features. Although the system proposed by Long was not used to simulate the nonlinear properties of the crow, the additional linkage somewhat contributed to slippage in the knee joint. Obviously, the three-link system cannot express the slippage directly. Experiments in the next section show that the chaotic component of the three-link system is not significant.

In the model shown in Figure 2 (c), the eccentric cam acts as the drive cam, and the rotational center of the other eccentric cam 02 moves around the cam surface of the drive cam O1. Obviously, the motion curve of $\mathrm{O} 2$ relative to the $\mathrm{O} 1$ cam surface is consistent with the cosine curve, ensuring smooth movement. It is not difficult to see that when the moving wheel $\mathrm{O} 1$ is moving periodically, the moving center of the eccentric wheel $\mathrm{O} 2$ moves periodically around the surface of the moving wheel, and the eccentric wheel $\mathrm{O} 2$ also rotates accordingly. These three periodic motions are mixed together and can easily excite the chaotic vibrations of the system. Then, the chaotic dynamics of the system is analyzed by modeling the dynamics of the system. 


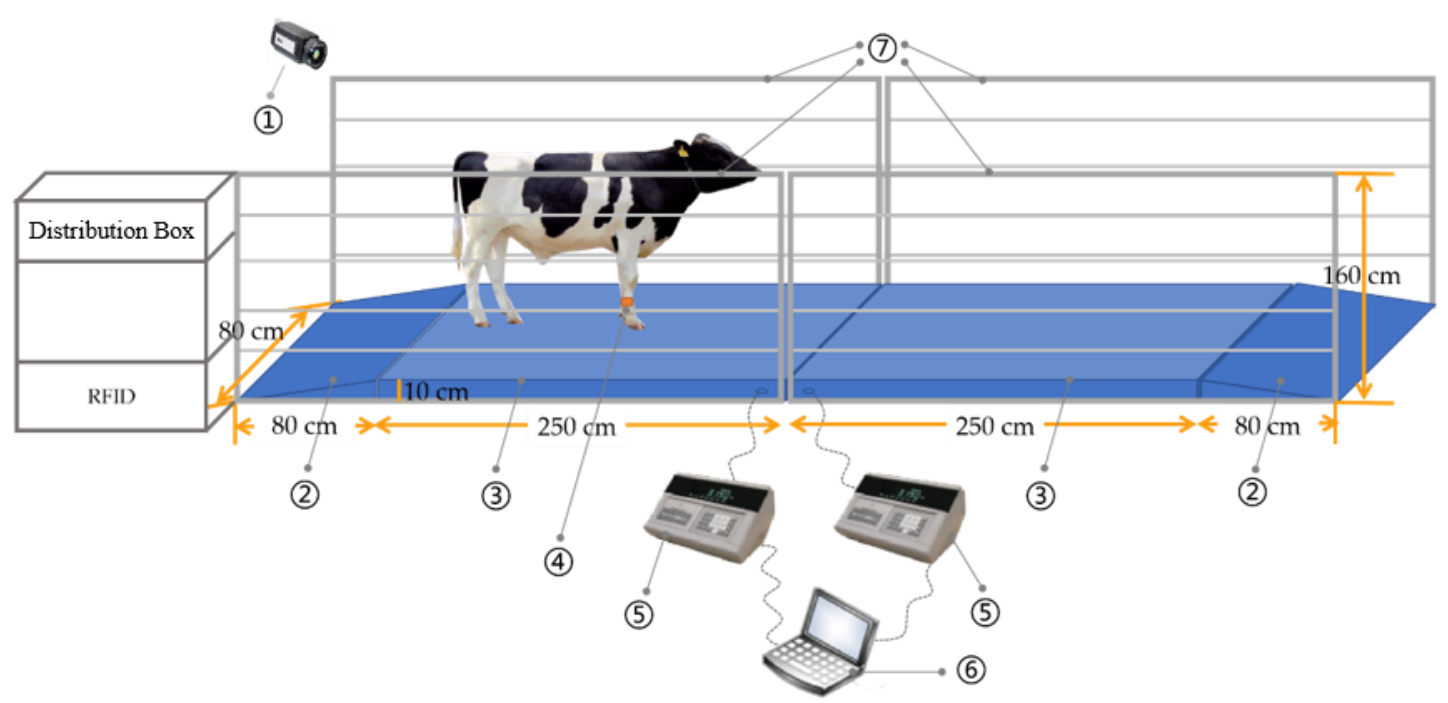

Fig.1 Pressure sensitive channel

1-Camera; 2-Slope; 3-Pressure sensors platform; 4-anklet; 5-Pressure gauge; 6-Computer; 7-Fence

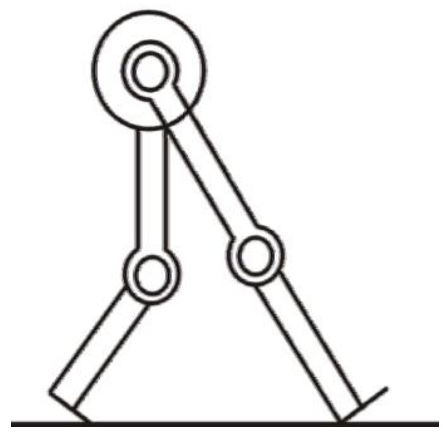

(a) two-link model

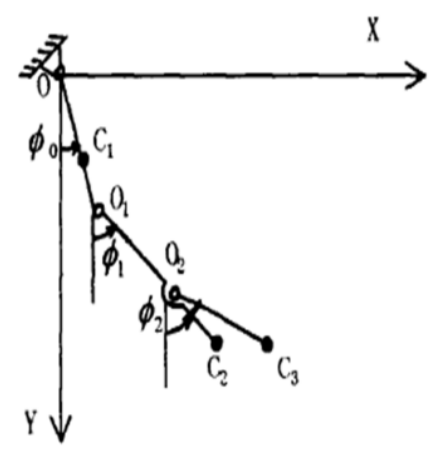

(b) three-link model

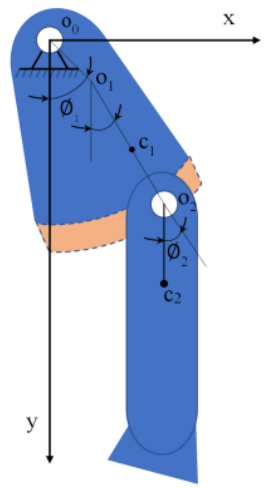

(c) two-link with sliding

Fig.2 Dynamic system of Cow Leg

Let's say $\mathrm{m} 1$ is the mass of eccentric I. Its moment of inertia is $J_{C_{1}}=\frac{1}{4} m_{1} R_{1}^{2}$ to the center of mass $\mathrm{C} 1(\mathrm{xc} 1, \mathrm{yc} 1)$. The angular velocity of eccentric I is denoted as $\omega$, the eccentricity 11 , and the radius $\mathrm{R} 1$. For eccentric II, M2 is the mass, and the moment of inertia is $J_{C_{2}}=\frac{1}{4} m_{2} R_{2}^{2}$ to the center of mass $C_{2}\left(x_{c 2}, y_{c 2}\right)$. The velocities of chain $O_{2}\left(x_{o 2}, y_{o 2}\right)$ and center of gravity $C_{2}$ are $V_{O 2}$ and $V_{C 2}$, respectively, and the eccentricity distance and radius are $l_{2}$ and $R_{2}$ respectively. The above parameters satisfy the following relationship:

$$
\begin{aligned}
& x_{C_{1}}=l_{1} \sin \left(\phi_{0}\right) \\
& \dot{x}_{C_{1}}=l_{1} \omega \cos \left(\phi_{0}\right) \\
& y_{C_{1}}=l_{1} \cos \left(\phi_{0}\right) \\
& \dot{y}_{C_{1}}=-l_{1} \omega \sin \left(\phi_{0}\right) \\
& x_{O_{2}}=x_{C_{1}}+R_{1} \sin \left(\phi_{1}\right) \\
& \dot{x}_{O_{2}}=\dot{x}_{C_{1}}+R_{1} \dot{\phi}_{1} \cos \left(\dot{\phi}_{1}\right)
\end{aligned}
$$

$$
\begin{aligned}
& y_{O_{2}}=y_{C_{1}}+R_{1} \cos \left(\phi_{1}\right) \\
& \dot{y}_{O_{2}}=\dot{y}_{C_{1}}-R_{1} \dot{\phi}_{1} \sin \left(\phi_{1}\right) \\
& x_{C_{2}}=x_{o_{2}}+l_{2} \sin \left(\phi_{2}\right) \\
& \dot{x}_{C_{2}}=\dot{x}_{o_{2}}+l_{2} \dot{\phi}_{2} \cos \left(\phi_{2}\right) \\
& y_{C_{2}}=y_{o_{2}}+l_{2} \cos \left(\phi_{2}\right) \\
& \dot{y}_{C_{2}}=\dot{y}_{o_{2}}-l_{2} \dot{\phi}_{2} \sin \left(\phi_{2}\right)
\end{aligned}
$$

The velocity of the hinge $\mathrm{O}_{2}$ is defined as 


$$
\begin{aligned}
& V_{O_{2}}^{2}=\left(\dot{x}_{O_{2}}\right)^{2}+\left(\dot{y}_{O_{2}}\right)^{2}=\left(\dot{x}_{C_{1}}+R_{1} \dot{\phi}_{1} \cos \left(\phi_{1}\right)\right)^{2}+\left(\dot{y}_{C_{1}}-R_{1} \dot{\phi}_{1} \sin \left(\phi_{1}\right)\right)^{2} \\
& =\left(l_{1} \omega\right)^{2}+\left(R_{1} \dot{\phi}_{1}\right)^{2}+2 \dot{\phi}_{1} l_{1}\left(\dot{x}_{C_{1}} \cos \left(\phi_{1}\right)-\dot{y}_{C_{1}} \sin \left(\phi_{1}\right)\right)=\left(l_{1} \omega\right)^{2}+\left(R_{1} \dot{\phi}_{1}\right)^{2}+2 \dot{\phi}_{1} l_{1} l_{2} \omega \cos \left(\omega t-\phi_{1}\right)
\end{aligned}
$$

The velocity of the centroid $C_{2}$

$$
\begin{aligned}
& \left(V_{C_{2}}\right)^{2}=\left(\dot{x}_{C_{2}}\right)^{2}+\left(\dot{y}_{C_{2}}\right)^{2} \\
& =\left(l_{1} \omega \cos \left(\phi_{0}\right)+R_{1} \dot{\phi}_{1} \cos \left(\phi_{1}\right)+l_{2} \dot{\phi}_{2} \cos \left(\phi_{2}\right)\right)^{2}+\left(-l_{1} \omega \sin \left(\phi_{0}\right)-R_{1} \dot{\phi}_{1} \sin \left(\phi_{1}\right)-l_{2} \dot{\phi}_{2} \sin \left(\phi_{2}\right)\right)^{2} \\
& =\left(V_{O_{2}}\right)^{2}+\left(l_{2} \dot{\phi}_{2}\right)^{2}+2 l_{1} l_{2} \dot{\phi}_{2} \omega \sin \left(\phi_{0}-\phi_{2}\right)+2 R_{1} l_{2} \dot{\phi}_{1} \dot{\phi}_{2} \cos \left(\phi_{1}-\phi_{2}\right)
\end{aligned}
$$

The kinetic energy of the system can be calculated as follows

1. The eccentric wheel I rotates as a fixed axis, the kinetic energy is

$T_{1}=\frac{1}{2} m_{1}\left(l_{1} \omega\right)^{2}+\frac{1}{2} J_{C_{1}} \omega^{2}=\frac{1}{8} m_{1} \omega^{2}\left(4 l_{1}^{2}+R_{1}^{2}\right)$

2. The eccentric wheel II do the plane movement, the kinetic energy is

$$
\begin{aligned}
T_{2}= & \frac{1}{2} m_{2}\left(V_{C_{2}}\right)^{2}+\frac{1}{2} J_{C_{2}}\left(\dot{\phi}_{2}\right)^{2}=\frac{1}{2} m_{2}\left[\left(l_{1} \omega\right)^{2}+\left(R_{1} \dot{\phi}_{1}\right)^{2}+2 \dot{\phi}_{1} l_{1} l_{2} \omega \cos \left(\omega t-\phi_{1}\right)+\left(l_{2} \dot{\phi}_{2}\right)^{2}\right. \\
& \left.+2 l_{1} l_{2} \dot{\phi}_{2} \omega \sin \left(\phi_{0}-\phi_{2}\right)+2 R_{1} l_{2} \dot{\phi}_{1} \dot{\phi}_{2} \cos \left(\phi_{1}-\phi_{2}\right)\right]+\frac{1}{8} m_{2}\left(R_{2} \dot{\phi}_{2}\right)^{2}
\end{aligned}
$$

The Total kinetic energy is defined as

$$
\begin{aligned}
& T=T_{1}+T_{2}=\frac{1}{2} m_{2}\left[\left(l_{1} \omega\right)^{2}+\left(R_{1} \dot{\phi}_{1}\right)^{2}+2 \dot{\phi}_{1} l_{1} l_{2} \omega \cos \left(\omega t-\phi_{1}\right)+\left(l_{2} \dot{\phi}_{2}\right)^{2}+2 l_{1} l_{2} \dot{\phi}_{2} \omega \sin \left(\phi_{0}-\phi_{2}\right)+\right. \\
& \left.+2 R_{1} l_{2} \dot{\phi}_{1} \dot{\phi}_{2} \cos \left(\phi_{1}-\phi_{2}\right)\right]+\frac{1}{8}\left[m_{1} \omega^{2}\left(4 l_{1}^{2}+R_{1}^{2}\right)+m_{2}\left(R_{2} \dot{\phi}_{2}\right)^{2}\right]
\end{aligned}
$$

Next, we calculate the potential energy of the system.

The potential energy of the system is 0 as $\phi_{0}=\phi_{1}=\phi_{2}=0$. In other cases, the potential energy of the system is

$$
U_{1}=m_{1} g l_{1}(1-\cos (\omega t))
$$

The potential energy of the eccentric wheel II is

$$
U_{2}=m_{2} g\left[l_{1}(1-\cos (\omega t))+R_{1}\left(1-\cos \left(\phi_{1}\right)\right)+l_{2}\left(1-\cos \left(\phi_{2}\right)\right)\right]
$$

The total potential energy of the system is

$$
\begin{aligned}
U & =U_{1}+U_{2}=m_{1} g l_{1}(1-\cos (\omega t))+m_{2} g\left[l_{1}(1-\cos (\omega t))+R_{1}\left(1-\cos \left(\varnothing_{1}\right)\right)+l_{2}\left(1-\cos \left(\varnothing_{2}\right)\right)\right] \\
& =\left(m_{1}+m_{2}\right) g l_{1}(1-\cos (\omega t))+m_{2} g\left[R_{1}\left(1-\cos \left(\varnothing_{1}\right)\right)+l_{2}\left(1-\cos \left(\varnothing_{2}\right)\right)\right]
\end{aligned}
$$

The Laplace function is defined as

$$
\begin{aligned}
L= & T-U=\frac{1}{2} m_{2}\left[\left(l_{1} \omega\right)^{2}+\left(R_{1} \dot{\phi}_{1}\right)^{2}+2 \dot{\phi}_{1} l_{1} l_{2} \omega \cos \left(\omega t-\phi_{1}\right)+\left(l_{2} \dot{\phi}_{2}\right)^{2}+2 l_{1} l_{2} \dot{\phi}_{2} \omega \sin \left(\phi_{0}-\phi_{2}\right)+\right. \\
& \left.2 R_{1} l_{2} \dot{\phi}_{1} \dot{\phi}_{2} \cos \left(\phi_{1}-\phi_{2}\right)\right]
\end{aligned}
$$

Take the Laplace function into the second kind of Lagrange equation, we obtain 


$$
\frac{d}{d t}\left(\frac{\partial L}{\partial \dot{\phi}_{i}}\right)-\frac{\partial L}{\partial \phi_{i}}=0, \quad i=1,2
$$

Where,

$$
\begin{aligned}
& \frac{\partial L}{\partial \dot{\phi}_{1}}=m_{2}\left(R_{1}\right)^{2} \dot{\phi}_{1}+m_{2} l_{1} R_{1} \omega \cos \left(\omega t-\phi_{1}\right)+m_{2} R_{1} l_{2} \dot{\phi}_{2} \cos \left(\phi_{1}-\phi_{2}\right) \\
& \frac{\partial L}{\partial \dot{\phi}_{2}}=\frac{1}{4} m_{2}\left(\left(2 l_{2}\right)^{2}+\left(R_{2}\right)^{2}\right) \dot{\phi}_{2}+m_{2} l_{1} l_{2} \omega \sin \left(\omega t-\phi_{2}\right)+m_{2} R_{1} l_{2} \dot{\phi}_{1} \cos \left(\phi_{1}-\phi_{2}\right) \\
& \frac{d}{d t}\left(\frac{\partial L}{\partial \dot{\phi}_{1}}\right)=m_{2}\left(R_{1}\right)^{2} \ddot{\phi}_{1}-m_{2} l_{1} R_{1} \omega\left(\omega-\dot{\phi}_{1}\right) \sin \left(\omega t-\phi_{1}\right)+m_{2} l_{2} R_{1} \cos \left(\phi_{1}-\phi_{2}\right) \ddot{\phi}_{2}- \\
& m_{2} l_{2} R_{1} \dot{\phi}_{2}\left(\dot{\phi}_{1}-\dot{\phi}_{2}\right) \sin \left(\phi_{1}-\phi_{2}\right) \\
& \frac{d}{d t}\left(\frac{\partial L}{\partial \dot{\phi}_{2}}\right)=\frac{1}{4} m_{2}\left(\left(2 l_{2}\right)^{2}+\left(R_{2}\right)^{2}\right) \ddot{\phi}_{2}+m_{2}\left(R_{1}\right)^{2} \ddot{\phi}_{1}-m_{2} l_{1} l_{2} \omega\left(\omega-\dot{\phi}_{2}\right) \cos \left(\omega t-\phi_{2}\right)+ \\
& +m_{2} l_{2} R_{1} \cos \left(\phi_{1}-\phi_{2}\right) \ddot{\phi}_{1}-m_{2} l_{2} R_{1} \dot{\phi}_{1}\left(\dot{\phi}_{1}-\dot{\phi}_{2}\right) \sin \left(\phi_{1}-\phi_{2}\right) \\
& \frac{\partial L}{\partial \phi_{1}}=-m_{2} g R_{1} \sin \left(\phi_{1}\right)+m_{2} l_{1} R_{1} \omega \dot{\phi}_{1} \sin \left(\omega t-\phi_{1}\right)-m_{2} l_{2} R_{1} \dot{\phi}_{1} \dot{\phi}_{2} \sin \left(\phi_{1}-\phi_{2}\right) \\
& \frac{\partial L}{\partial \phi_{2}}=-m_{2} g l_{2} \sin \left(\phi_{2}\right)-m_{2} l_{1} l_{2} \omega \dot{\phi}_{2} \cos \left(\omega t-\phi_{2}\right)+m_{2} l_{2} R_{1} \dot{\phi}_{1} \dot{\phi}_{2} \sin \left(\phi_{1}-\phi_{2}\right)
\end{aligned}
$$

Thus, the second kind of Lagrange equation can be rewritten as the nonlinear system of ordinary differential equations as follows

$$
\left[\begin{array}{ll}
a_{11} & a_{12} \\
a_{21} & a_{22}
\end{array}\right]\left\{\begin{array}{l}
\ddot{\phi}_{1} \\
\ddot{\phi}_{2}
\end{array}\right\}=\left\{\begin{array}{l}
b_{1} \\
b_{2}
\end{array}\right\}
$$

where

$$
\begin{aligned}
a_{11} & =m_{2}\left(R_{1}\right)^{2}, a_{12}=m_{2} l_{2} R_{1} \cos \left(\phi_{1}-\phi_{2}\right), a_{21}=a_{12}, a_{22}=\frac{1}{4} m_{2}\left(\left(2 l_{2}\right)^{2}+\left(R_{2}\right)^{2}\right) \\
b_{1}= & m_{2} l_{1} R_{1} \omega\left(\omega-\dot{\phi}_{1}\right) \sin \left(\omega t-\phi_{1}\right)+m_{2} l_{2} R_{1} \dot{\phi}_{2}\left(\dot{\phi}_{1}-\dot{\phi}_{2}\right) \sin \left(\phi_{1}-\phi_{2}\right)+ \\
& +m_{2} l_{1} R_{1} \omega \dot{\phi}_{1} \sin \left(\omega t-\phi_{1}\right)-m_{2} l_{2} R_{1} \dot{\phi}_{1} \dot{\phi}_{2} \sin \left(\phi_{1}-\phi_{2}\right)-m_{2} g R_{1} \sin \left(\phi_{1}\right) \\
& =m_{2} l_{1} R_{1} \omega^{2} \sin \left(\omega t-\phi_{1}\right)-m_{2} l_{2} R_{1}\left(\dot{\phi}_{2}\right)^{2} \sin \left(\phi_{1}-\phi_{2}\right)-m_{2} g R_{1} \sin \left(\phi_{1}\right) \\
b_{2}= & -m_{2} l_{1} l_{2} \omega\left(\omega-\dot{\phi}_{2}\right) \cos \left(\omega t-\phi_{2}\right)-m_{2} l_{1} l_{2} \omega \dot{\phi}_{2} \cos \left(\omega t-\phi_{2}\right)+m_{2} l_{1} R_{1} \dot{\phi}_{1}\left(\dot{\phi}_{1}-\dot{\phi}_{2}\right) \sin \left(\phi_{1}-\phi_{2}\right)+ \\
& +m_{2} l_{2} R_{1} \dot{\phi}_{1} \dot{\phi}_{2} \sin \left(\phi_{1}-\phi_{2}\right)-m_{2} g l_{2} \sin \left(\phi_{2}\right)
\end{aligned}
$$

Eq.(1) is the dynamic system of the proposed model of dairy cow leg.

\section{Dynamics Simulation}

Qualitative and quantitative methods are usually used to judge whether the system vibration has chaotic components. In the qualitative method, the phase orbit diagram of nonlinear dynamic model is often taken as the judge tools. The phase orbit diagram of chaotic vibration system has the characteristics 
of non repetition, irregularity and repeated winding; The typical quantitative method is by mean of the Lyapunov index, we think the system can produce the chaotic vibration as the maximum of the Lyapunov index is greater than 0 .

Qualitative and quantitative methods are often used to determine whether chaotic components exist in system vibrations. In qualitative methods, phase diagrams of nonlinear dynamic models are often used as a judgment tool. The phase diagram of chaotic vibrational systems is characterized by non-repetitive, irregular, and derived replications. A typical quantitative method is to use the Lyapunov scale. We argue that the system may experience chaotic vibrations when the maximum of the Lyapunov exponent is greater than 0 .

2.1 Phase orbit diagram method Let

$$
A=\left|\begin{array}{ll}
a_{11} & a_{12} \\
a_{21} & a_{22}
\end{array}\right| \quad B_{1}=\left|\begin{array}{ll}
b_{1} & a_{12} \\
b_{2} & a_{22}
\end{array}\right| \quad B_{2}=\left|\begin{array}{ll}
a_{11} & b_{1} \\
a_{21} & b_{2}
\end{array}\right|
$$

The solution of above ODE can be expressed as

$$
\ddot{\phi_{1}}=B_{1} / A \quad \ddot{\phi}_{2}=B_{2} / A
$$

In simulation, the masses of the two cams are $m_{1}=120, m_{2}=129$ respectively; $l_{0}=0.00033464 ; l_{1}=0.0039$; $l_{2}=0.0178 ; R=0.02$. As can be seen from Fig. 3 , with the increase of the angular velocity of the driving wheel, the phase orbit diagram of the system becomes more and more complex, and the chaotic components become more and more. To some extent, it also reflects the process of the system from periodic motion to chaos.

2.2 Calculation of the maximum of the Lyapunov index $\lambda_{\max }$

There are many method to calculate $\lambda_{\max }$,such as the definition method, Orthogonalization method, wolf method, and so on. Here we take the definition method to calculate it. Let $y_{1}=\phi_{1}, y_{2}=\phi_{2}, y_{3}=\dot{\phi}_{1}, y_{4}=\dot{\phi}_{2}, f_{3}(y)=B_{1} / A, f_{4}(y)=B_{2} / A$

where $y=\left[\begin{array}{llll}y_{1} & y_{2} & y_{3} & y_{4}\end{array}\right]^{T}$, then, the nonlinear system of ODEs can be expressed as

$$
\left\{\begin{array}{l}
\dot{y}_{1}=y_{3} \\
\dot{y}_{2}=y_{4} \\
\dot{y}_{3}=f_{3}(y) \\
\dot{y}_{4}=f_{4}(y)
\end{array}\right.
$$

The corresponding Jacobi matrix at any point can be expressed as

$$
J a c=\left|\begin{array}{clll}
0 & 0 & 1 & 0 \\
0 & 0 & 0 & 1 \\
\frac{\partial f_{3}}{\partial y_{1}} & \frac{\partial f_{3}}{\partial y_{2}} & \frac{\partial f_{3}}{\partial y_{3}} & \frac{\partial f_{3}}{\partial y_{4}} \\
\frac{\partial f_{4}}{\partial y_{1}} & \frac{\partial f_{4}}{\partial y_{2}} & \frac{\partial f_{4}}{\partial y_{3}} & \frac{\partial f_{4}}{\partial y_{4}}
\end{array}\right|
$$

The largest Lyapunov exponent curve respect to time calculated by above method is showed in Fig.3. The Lyapunov index of the proposed system approximately equals to 7 , which is greater than the 3-link system significantly. Obviously, the proposed system is a more valuable dairy cow leg model. 


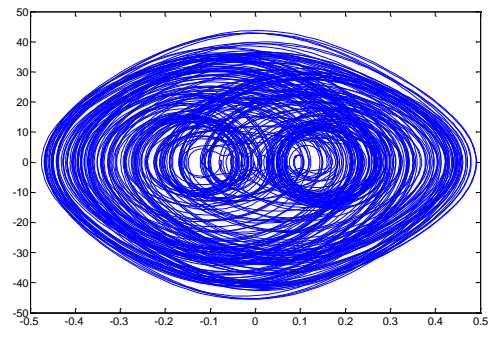

$w=60$

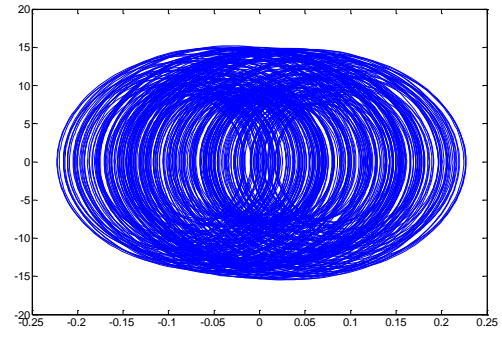

$w=40$

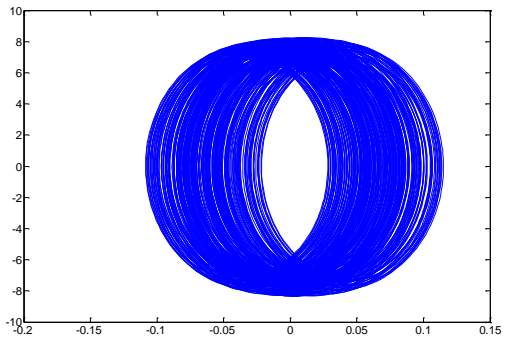

$w=20$

Fig.3 Variation of the system phase orbit diagram with the rotational angular velocity of the driving wheel

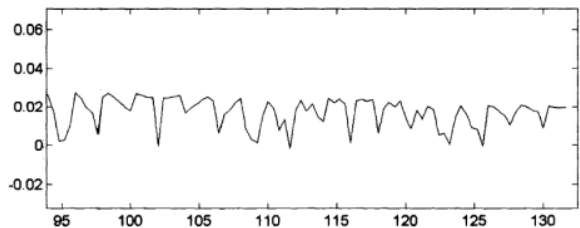

(a) Three-link system

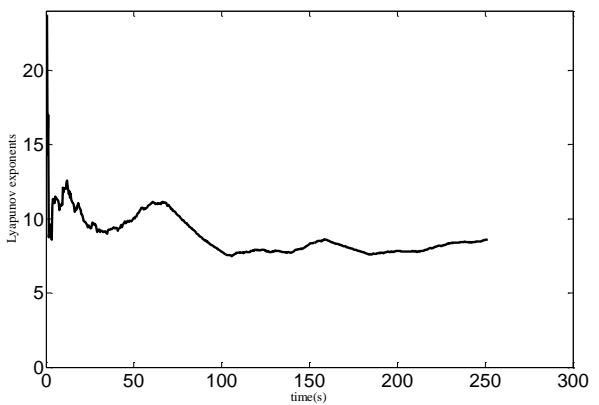

(b) Proposed system

Fig. 4 The largest Lyapunov exponent curve respect to time

\section{Conclusions}

In the analysis, we can see that a new chaotic vibration system based on the relationship between the branch overlapping period and the chaotic phenomenon is more chaotic and the parameter selection of the chaotic vibration system is relatively loose. Three links are studied, the eccentric motion is stable, the vibration and shock are small, the structure And the processing technology is simple, easy to handle and realize. In addition, the qualitative analysis theory and method theory are used to analyze the overall structure optimization and system parameters of the dynamic system, and find the best value that comprehensively considers the resistance mechanical components and the ghost of the system components.

\section{References}

[1] J.C.Zaffino Heyerhoff, S.J.LeBlanc, T.J.DeVries, C.G.R. Nash, J.Gibbons, K.Orsel, H.W.Barkema, L.Solano, J.Rushen, A.M.de Passille, and D.B.Haley. Prevalence of and factors associated with hock, knee, and neck injuries on dairy cows in freestall housing in Canada. J.Dairy Sci. 97(2014):173-184

[2] Ningning Feng, Xi Kang, Haoyuan Han, Gang Liu, Yan'e Zhang*, and Shuli Mei. Research on a Dynamic Algorithm for Cow Weighing Based on an SVM and Empirical Wavelet Transform. Sensors 2020, 20, 5363; doi:10.3390/s20185363

[3] Andreas Merker, Dieter Kaiser, Andre Seyfarth, and Martin Hermann. Stable running with asymmetric legs: a bifurcation approach. International Journal of Biuration and Chaos, Vol.25,No.11(2015) 1550152

[4] Long Yunjia, Liang Yide. Modren Engineering Dynamics-Random \& Chaos [M]. Science Press, 1998 (in Chinese with English abstract)

[5] Xing Ruyi, Yang Yong. A New Chaotic Exciter and Its Dynamic Characteristics. Journal of China Agricultural Unviersity, vol.19,No.01(2014):175-179 